\title{
Generalized Romberg Methods for Integrals of Derivatives
}

\author{
J. N. LYNESS * and C. B. MOLER *夫 \\ Received September 19, 1967
}

\section{Introduction}

Certain algorithms for calculating the Fourier coefficients of a smooth function $f(x)$ (Stetter [7], Lyness [4]) require approximate values of the integrals of $f$ and some of its derivatives. We denote these by

$$
\begin{aligned}
& I_{0}^{[1]}=\int_{0}^{1} f(x) d x \\
& I_{s}^{[1]}=\int_{0}^{1} f^{(s)}(x) d x=f^{(s-1)}(1)-f^{(s-1)}(0), \quad s=1,2, \ldots
\end{aligned}
$$

One method for calculating these quantities can be based on the EulerMaclaurin summation formula:

$$
\begin{aligned}
\frac{1}{m}\left[\frac{1}{2} f(0)+\right. & \left.f\left(\frac{1}{m}\right)+f\left(\frac{2}{m}\right)+\cdots+f\left(\frac{m-1}{m}\right)+\frac{1}{2} f(1)\right] \\
= & I_{0}^{[1]}+\frac{d_{2}}{m^{2}} I_{2}^{[1]}+\frac{d_{2}}{m^{4}} I_{4}^{[1]}+\cdots+\frac{d_{p}}{m^{2 p}} I_{2 p}^{[1]} \\
& +\frac{2(-1)^{p}}{(2 \pi m)^{2 p+2}} \int_{0}^{1} f^{(2 p+2)}(x) \sum_{r=1}^{\infty} \frac{1-\cos 2 \pi r m x}{r^{2 p+2}} d x
\end{aligned}
$$

where $m$ and $p$ are arbitary integers and $d_{s}$ are certain known constants independent of both $m$ and $p$. The term on the left is the trapezoidal rule applied to $f(x)$ and the final term on the right is referred to as the remainder term.

If the trapezoidal rule is evaluated for $p+1$ distinct values of $m$ and the remainder term is ignored, then (1.2) forms a set of $p+1$ linear equations, which may be solved to obtain approximations to the $p+1$ unknowns

$$
I_{0}^{[1]}, I_{2}^{[1]}, \ldots, I_{2 p}^{[1]} .
$$

The approximation to $I_{0}^{[1]}$ thus obtained is exactly the same as that obtained by $p+1$ steps of Romberg integration.

Expansions analogous to (1.2) involving displaced trapezoidal rule and combinations of these may be employed in an identical manner to determine approximations to $I_{s}$ for odd $s$ and other approximations to $I_{s}$ for even $s$. The purpose

* Present address: Applied Mathematics Division, Argonne National Laboratory, Argonne, Illinois 60439 .

* Present address: Department of Mathematics, University of Michigan, Ann Arbor, Michigan 48104. 
of this paper is to discuss these modifications, describe Romberg-like algorithms for the solution of the resulting linear equations and analyze the truncation error which arises from ignoring the remainder term.

\section{The Basic Operators and Their Euler-Maclaurin Expansions}

The familiar end point trapezoidal quadrature rule may be described in operator notation by $R^{[m, 1]} f(x)$ where

$$
\begin{aligned}
R^{[m, 1]} f(x) & =\frac{1}{m} \sum_{j=0}^{m} f\left(\frac{j}{m}\right) \\
& \equiv \frac{1}{m}\left(\frac{1}{2} f(0)+f\left(\frac{1}{m}\right)+f\left(\frac{2}{m}\right)+\cdots+f\left(\frac{m-1}{m}\right)+\frac{1}{2} f(1)\right) .
\end{aligned}
$$

The displaced trapezoidal quadrature rule is defined by

$$
R^{[m, \alpha]} f(x)=\frac{1}{m} \sum_{j=1}^{m} f\left(\frac{2 j-1+\alpha}{2 m}\right), \quad|\alpha|<1 .
$$

The superscript $m$ defines the number of panels which are of equal length and the superscript $\alpha$ the relative displacement of the point for function evaluation from the centre of each panel. An expansion similar to (1.2) exists for the displaced trapezoidal quadrature rule. This is

$$
R^{[m, \alpha]} f(x)=\sum_{s=0}^{w} \frac{c_{s}\left(t_{a}\right)}{m^{s}} \int_{0}^{1} f^{(s)}(x) d x+\frac{1}{m^{w+1}} \int_{0}^{1} g_{w+1}\left(m x, t_{\alpha}\right) f^{(w+1)}(x) d x
$$

where

$$
\begin{aligned}
& t_{\alpha}=\frac{\alpha+1}{2}, \\
& c_{s+1}(t)=-\frac{\zeta(-s, t)}{s !}=\frac{B_{s+1}(t)}{(s+1) !}, \quad s=0,1,2, \ldots
\end{aligned}
$$

and the function $g_{w+1}(x, t)$ which is periodic with period 1 is given by

$$
\begin{aligned}
g_{w+1}(x, t) & =[\bar{\zeta}(-w, t-x)-\zeta(-w, t)] / w ! \\
& =\left[\bar{B}_{w+1}(t-x)-B_{w+1}(t)\right] /(w+1) ! .
\end{aligned}
$$

Here $\zeta(s, t)$ is the generalized zeta function and $B_{s}(t)$ the Bernoulli polynomial. The bars on these functions indicate the corresponding periodic functions, which coincide with the respective functions in the interval $0<t<1$. The notation adopted for these functions is that of Abramowitz and Stegun [1] and properties of these functions listed there will be assumed without proof.

In this paper we are interested in four distinct operators. The first two are $R^{[m, 0]} f(x)$ and $R^{[m, 1]} f(x)$, which Stetter [7] refers to as the rectangular sum and the trapezoidal sum. These have expansions in which the terms in odd powers of $m$ are zero (as in (1.2)) and the remainder terms of even order ( $w+1$ even) contain weighting functions $g_{w+1}\left(m x, t_{\alpha}\right)$ which are of definite sign when considered as a function of $x$. The third is the alternating trapezoidal sum

$$
R^{[2 m, 1]}(f(x) \cos 2 \pi m x)=\frac{1}{2}\left[R^{[m, 1]} f(x)-R^{[m, 0]} f(x)\right]
$$


which has the same properties, and the additional property that the constant term in the expansion is zero. Finally we consider the alternating rectangular sum

$$
R^{[2 m, 0]}(f(x) \sin 2 \pi m x)=\frac{1}{2}\left[R^{\left[m,-\frac{1}{2}\right]} f(x)-R^{\left[m, \frac{1}{2}\right]} f(x)\right] .
$$

The expansion corresponding to this operator contains only odd inverse powers of $m$, and the remainder term of odd order is of definite sign.

The results of this paper apply equally to all four operators. It is convenient to redefine these operators in such a way that all have even expansions, starting with a non-zero constant term. We define

$$
\begin{aligned}
& P^{[m, 0]} f(x)=R^{[m, 0]} f(x) \\
& P^{[m, 1]} f(x)=R^{[m, 1]} f(x) \\
& P^{[m, 2]} f(x)=m^{2} R^{[2 m, 1]}(f(x) \cos 2 \pi m x) \\
& P^{[m, 3]} f(x)=m R^{[2 m, 0]}(f(x) \sin 2 \pi m x) .
\end{aligned}
$$

The expansion for each of these operators may be written in the form

$$
\begin{aligned}
P^{[m, \alpha]} f(x)= & \sum_{s=0}^{p} \frac{d_{s}^{[\alpha]}}{m^{2 s}} \int_{0}^{1} \frac{d^{2 s}}{d x^{2 s}} \phi^{[\alpha]}(x) d x \\
& +\frac{1}{m^{2 p+2}} \int_{0}^{1} h_{p+1}^{[\alpha]}(m x) \frac{d^{2 p+2}}{d x^{2 p+2}} \phi^{[\alpha]}(x) d x
\end{aligned}
$$

where $d_{s}^{[\alpha]}$ and $h_{p+1}^{[\alpha]}(x)$ are constants and functions involving zeta functions and associated functions given below and $\phi^{[\alpha]}(x)$ stands for $f(x)$ or one of its derivatives. The remainder of this section is concerned only with stating expressions for these constants and functions for different values of $\alpha$.

For $\alpha=0,1,2,3$ we have

$$
\begin{aligned}
& \phi^{[0]}(x)=\phi^{[1]}(x)=f(x) \\
& \phi^{[2]}(x)=f^{\prime \prime}(x) \\
& \phi^{[3]}(x)=f^{\prime}(x)
\end{aligned}
$$

and we define in analogy to (1.1)

$$
I_{2 s}^{[\alpha]}=\int_{0}^{1} \frac{d^{2 s} \phi^{[\alpha]}(x)}{d x^{2 s}} d x
$$

The constants $d_{s}^{[\alpha]}$ are given by

$$
\begin{aligned}
& d_{s}^{[0]}=\frac{2(-1)^{s+1}}{(2 \pi)^{2 s}} \sum_{r=1}^{\infty} \frac{(-1)^{r}}{r^{2 s}}=-\frac{2(-1)^{s+1}}{(2 \pi)^{2 s}} \eta(2 s) \\
& d_{s}^{[1]}=\frac{2(-1)^{s+1}}{(2 \pi)^{2 s}} \sum_{r=1}^{\infty} \frac{1}{r^{2 s}}=\frac{2(-1)^{s+1}}{(2 \pi)^{2 s}} \zeta(2 s) \\
& d_{s}^{[2]}=\frac{2(-1)^{s}}{(2 \pi)^{2 s+2}} \sum_{r \text { odd }} \frac{1}{r^{2 s+2}}=\frac{2(-1)^{s}}{(2 \pi)^{2 s+2}} \lambda(2 s+2) \\
& d_{s}^{[3]}=\frac{2(-1)^{s+1}}{(2 \pi)^{2 s+1}} \sum_{r \text { odd }} \frac{(-1)^{(r-1) / 2}}{r^{2 s+1}}=\frac{2(-1)^{s+1}}{(2 \pi)^{2 s+1}} \beta(2 s+1)
\end{aligned}
$$


The functions $\eta(s), \zeta(s), \lambda(s)$ and $\beta(s)$ are tabulated in [1], pp. 811-812. Simple relations exist connecting the first three, which may be expressed in terms of Bernoulli numbers. $\beta(2 s+1)$ may be expressed simply in terms of Euler numbers.

The functions $h_{p+1}^{[\alpha]}(x)$ are the appropriate linear combinations of $g_{t w+1}(x, t)$ with $t=0, \frac{1}{4}, \frac{1}{2}, \frac{3}{4}, 1$. These functions are periodic with period 1. The Fourier expansion of $h_{p+1}^{[\alpha]}(x)$ is given by

$$
h_{p+1}^{[\alpha]}(x)=\frac{4(-1)^{p+1}}{(2 \pi)^{2 p+2}} \sum_{i=1}^{\infty} \frac{N_{t}^{[\alpha]} \sin ^{2} \pi x x}{\gamma^{2 p+2}}
$$

where

$$
\begin{aligned}
N_{r}^{[0]} & =(-1)^{r+1} \\
N_{r}^{[1]} & =-1 \\
N_{2 r}^{[2]} & =1 /(8 \pi r)^{2} \\
N_{2 r+1}^{[2]} & =0 \\
N_{2 r}^{[3]} & =0 \\
N_{2 r+1}^{[3]} & =(-1)^{r+1} / 2 \pi(2 r+1) .
\end{aligned}
$$

It follows from $(2.10)$ directly that $d_{p+1}^{[\alpha]}$ is the mean value of $h_{p+1}^{[\alpha]}(x)$, that is

$$
a_{p+1}^{[\alpha]}=\int_{0}^{1} h_{p+1}^{[\alpha]}(m 2) d x \text { for any integer } m
$$

In the following sections, we suppress the superscript $[\alpha]$ in equations which are valid for $\alpha=0,1,2,3$.

\section{The Algorithm}

In this section we derive an algorithm for determining approximations to $d_{s}^{[\alpha]} I_{2 s}^{[\alpha]}$ using the procedure outlined in the introduction. Throughout the rest of this paper we suppose that we have a fixed set of distinct integers, the mesh ratios

$$
m_{0}, m_{1}, m_{2}, \ldots \text {, }
$$

and that we are dealing with a single operator $P^{[m, \alpha]}$, this being one of the operators defined in (2.9) and satisfying an Euler-Maclaurin expansion of type (2.10). We suppose that $P^{[m, \alpha]} f(x)$ has been evaluated numerically for $m=m_{k}, m_{k+1}, \ldots, m_{k+p}$ and we define

$$
T_{j, 0,0}=P^{[m, \alpha]} f(x)
$$

The Euler-Maclaurin expansion (2.10) has the form

$$
T_{i, 0,0}=\sum_{s=0}^{p} \frac{d_{s} I_{2 s}}{m_{j}^{2 s}}+\frac{1}{m_{j}^{2 p+2}} \int_{0}^{1} h_{p+1}\left(m_{j} x\right) \frac{d^{2 p+2}}{d x^{2 p+2}} \phi(x) d x .
$$

An approximation to $d_{s} I_{2 s}$ may be obtained by solving the set of $p+1$ linear equations obtained by setting $j=k, k+1, \ldots, k+p$ in (3.3) and ignoring the 
remainder term. This approximation $T_{k, p, s}$ is defined by

$$
\sum_{s=0}^{p} \frac{T_{k, p, s}}{m_{j}^{2 s}}=T_{i, 0,0}, \quad j=k, k+1, \ldots, k+p .
$$

The quantities $T_{k, p, 0}$ corresponding to the operator with $\alpha=1$, the trapezoidal rule are precisely the quantities $T_{p}^{(k)}$ discussed in Bauer, Rutishauser and Stiefel [2] and in Bulirsch [3]. These are higher order approximations to $I_{0}$ which occur in the $(p+1)$-th column of the Romberg Table. The quantities $T_{k, p, s}$ which are approximations to $d_{s} I_{2 s}$ form a three-dimensional array. This is a natural generalization of the Romberg Table, and we derive below an algorithm with which elements of this three-dimensional table may be calculated from neighboring elements.

The coefficient matrix $A^{[k, p]}$ of (3.4) is of course a $(p+1) \times(p+1)$ van der Monde matrix whose elements are given by

$$
\left(A^{[k, p]}\right)_{i_{3} j}=\left(a_{i+k}\right)^{j}, \quad i, j=0,1, \ldots, p \text {. }
$$

Here we have defined for convenience

$$
a_{j}=\frac{1}{m_{j}^{2}}
$$

An effective algorithm for solving such a system, which is a natural generalization of the Neville algorithm is discussed in Lyness and Moler [5]. In terms of the present notation, this algorithm may be written:

Algorithm (3.7)

$$
\begin{aligned}
& T_{k, 0,0}=P^{\left[m_{k}, \alpha\right]} f(x), \quad k=0,1, \ldots \\
& T_{k, p, s}=\frac{a_{k+p} T_{k, p-1, s}-a_{k} T_{k+1, p-1, s}+T_{k+1, p-1, s-1}-T_{k, p-1, s-1}}{a_{k+p}-a_{k}} \\
& p=1,2, \ldots, \quad s=0,1,2, \ldots, p
\end{aligned}
$$

where we define

$$
T_{k, p, s}=0, \quad s<0 \text { or } s>p .
$$

We note that if $s=0$, this is the conventional Romberg Algorithm. We also note that if approximations to $I_{0}, I_{2}, \ldots, I_{2 q}$ only are required, only $T_{k, p, s}$ for $s \leqq q$ need be calculated.

\section{The Truncation Error}

In this section we establish certain rather general results concerning the error caused by ignoring the remainder term. We refer to this error $T_{k, p, s}-d_{s} I_{2 s}$ as the truncation error. In order to do this it is convenient to derive certain results of an algebraic nature concerning the inverse of the van der Monde matrix (3.5) and the elementary symmetric functions of $a_{k}, a_{k+1}, \ldots, a_{k+p}$.

The elements of the matrix $\Gamma^{[k, p]}$ which is inverse to $A^{[k, p]}$ are denoted by

$$
\left(\Gamma^{[k, p]}\right)_{i, j}=\mu_{k, p, i, i+k}
$$


These have simple expressions in terms of the elements of $A^{[k, p]}$ but we do not require these expressions here. Since $\Gamma A=I$, it follows that

$$
\sum_{l=k}^{k+p} \mu_{k, p, i, l} a_{l}^{j}=\delta_{i, j}, \quad i, j=0,1, \ldots, p
$$

where $\delta_{i, j}$ is the Kronecker Delta. This equation is used many times throughout the rest of this paper. The solution of the set of linear equations (3.4) may be written

$$
T_{k, p, s}=\sum_{j=k}^{k+p} \mu_{k, p, s, j} T_{j, 0,0}, \quad s=0,1, \ldots, p .
$$

The elementary symmetric functions $\sigma_{k, p, l}$ of the numbers $a_{k}, a_{k+1}, \ldots, a_{k+p}$ may be defined as the coefficients of the monic polynomial which has roots $-a_{k},-a_{k+1}, \ldots,-a_{k+p}$. More precisely

In particular

$$
\prod_{t=k}^{k+p}\left(y-a_{t}\right) \equiv \sum_{j=0}^{p+1} \sigma_{k, p, j} y^{j}(-1)^{j+p+1}
$$

$$
\begin{aligned}
\sigma_{k, p, p+1} & =1 \\
\sigma_{k, p, p} & =a_{k}+a_{k+1}+\cdots+a_{k+p} \\
\cdot \cdot \cdot \cdot \cdot \cdot \cdot \cdot \cdot \cdot & \cdot \cdot \cdot \cdot a_{k+p} .
\end{aligned}
$$

A property of these numbers which we require later is that as $a_{i}>0$,

$$
\sigma_{k, p, l}>0 \text {. }
$$

These functions have a simple expression in terms of the elements of the corresponding van der Monde matrix and its inverse. This expression, (4.8) below, is classical in nature. A brief derivation is included here. The identity (4.4) may be written in the form

$$
y^{p+1} \equiv(-1)^{p} \sum_{j=0}^{p}(-1)^{i} \sigma_{k, p, j} y^{j}, \quad y=a_{k}, \ldots, a_{k+p} .
$$

We denote by $\sigma$ and $x$ the column vectors whose $j$-th elements are $(-1)^{j} \sigma_{k, p, j}$ and $(-1)^{p}\left(a_{k+j}\right)^{p+1}$ respectively, $j$ running from 0 to $p$. Abbreviating the appropriate van der Monde matrix (3.5) and its inverse (4.1) by $A$ and $\Gamma$ respectively, it follows from (4.6) that

$$
A \sigma=x .
$$

Since $\Gamma A=I$, this may be solved in the form $\sigma=\Gamma x$ which is expressed in components by the relations

$$
\sigma_{k, p, s}=(-1)^{s+p} \sum_{l=k}^{k+p} \mu_{k, p, s, l} a_{l}^{p+1}
$$

This relation is used later in this section.

We are now in a position to prove two theorems about the truncation error. 


\section{Theorem 1.}

$$
T_{k, p, s}-d_{s} I_{2 s}=\int_{0}^{1} b_{k, p, s}(x) \phi^{(2 p+2)}(x) d x
$$

where

$$
b_{k, p, s}(x)=\sum_{j=k}^{k+p} \mu_{k, p, s, j} a_{j}^{p+1} h_{p+1}\left(m_{j} x\right)
$$

Here $\phi^{(q)}(x)$ stands for the $q$-th derivative of $\phi(x)$, which is defined in (2.11) and is either $f(x), f^{\prime}(x)$ or $f^{\prime \prime}(x)$ depending on which of the four values of $\alpha$ is under consideration.

Proof. The Euler-Maclaurin expansion may be written

$$
T_{i, 0,0}=\sum_{l=0}^{p} d_{l} I_{2 l} a_{j}^{l}+a_{j}^{p+1} \int_{0}^{1} h_{p+1}\left(m_{j} x\right) \phi^{(2 p+2)}(x) d x .
$$

Using (4.3) above, we find

$$
T_{k, p, s}=\sum_{j=k}^{k+p} \mu_{k, p, s, j} \sum_{l=0}^{p} d_{l} I_{2 l} a_{j}^{l}+\int_{0}^{1} \sum_{j=k}^{k+p} \mu_{k, p, s, j} a_{j}^{p+1} h_{p+1}\left(m_{j} x\right) \phi^{(2 p+2)}(x) d x
$$

The double summation on the right hand side simplifies to $d_{s} I_{2 s}$ by the application of identity (4.2). The factor of $\phi^{(2 p+2)}(x)$ in the integral is by definition (4.10) simply $b_{k, p, s}(x)$. Consequently (4.12) reduces to (4.9) and Theorem 1 follows.

Theorem 2. If the function $b_{k, p, s}(x)$ defined by $(4.10)$ is of constant sign, then

$$
T_{k, p, s}-d_{s} I_{2 s}=(-1)^{p+s+1} \sigma_{k, p, s} d_{p+1} \phi^{(2 p+2)}(\xi)
$$

for some $\xi$ satisfying

$$
0 \leqq \xi \leqq 1
$$

Proof. Using the mean value theorem

$$
\int_{0}^{1} b_{k, p, s}(x) \phi^{(2 p+2)}(x) d x=\int_{0}^{1} b_{k, p s s}(x) d x \phi^{(2 p+2)}(\xi) .
$$

Since, by $(2.16)$

$$
\int_{0}^{1} h_{p+1}(m x) d x=d_{p+1}
$$

it follows using definition (4.10) and identity (4.8) that

$$
\begin{aligned}
\int_{0}^{1} b_{k, p, s}(x) d x & =\sum_{j=k}^{k+p} \mu_{k, p, s, j} a_{j}^{p+1} \int_{0}^{1} h_{p+1}\left(m_{j} x\right) d x \\
& =\sigma_{k, p, s} d_{p+1}(-1)^{p+s+1} .
\end{aligned}
$$

Theorem 2 follows from Theorem 1 using (4.15) and (4.17).

Theorem 2 expresses the truncation error in a remarkably simple form, in spite of the rather lengthy derivation. In general the value of $\phi^{(2 p+2)}(\xi)$ is unknown, but depends only on the function $f(x)$ and the particular operator $P^{[m, \alpha]}$ being used. The value of $d_{p+1}$ is a constant, related in a simple manner to the 
Bernoulli or Euler numbers, given by (2.13). The constant $\sigma_{k, p, s}$ depends only on the mesh ratios $m_{k}, m_{k+1}, \ldots, m_{k+p}$ and is the $s$-th symmetric function of the squares of their inverses, given by (4.4) or (4.5).

The difficulty in applying Theorem 2 lies in assuring oneself that the function $b_{k, p, s}(x)$ is of definite sign. The remainder of this paper is devoted to showing this in certain cases. This is determined by the choice of mesh ratios and the choice of operator $P^{[m, \alpha]}$. In the case of the trapezoidal rule $\alpha=1$ and mesh ratios $m_{i}=2^{i}$ Bauer, Rutishauser and Stiefel [2] have shown that $b_{k, p, 0}(x)$ (in their notation $\left.4^{-(p+1) k} b_{2 p+2}\left(2^{k} x\right)\right)$ is of definite sign. In the case of the trapezoidal rule $\alpha=1$ and mesh ratios $m_{i}=i+1$. Bulirsch [3] has shown that $b_{0, p, 0}(x)$ (in his notation $\left.(-1)^{p} S_{2 p+2}(x)\right)$ is of definite sign. In Section 6 we adapt the method given in Bulirsch [3] to show that for $\alpha=0,1,2,3$ and mesh ratios $m_{i}=i+1$, the functions $b_{0, p, s}(x)$ are of constant sign. Corresponding questions for other mesh ratios, operators and choices of $k, p$, and $s$ remain unanswered, except that Bulirsch [3] has also shown that for $\alpha=1$ and $m_{i}=i+1$, the function $b_{1, p, 0}(x)$ is not of constant sign.

\section{General Properties of $b_{k, p, s}(x)$}

Before restricting our attention to a particular set of mesh ratios, we briefly discuss the nature of the function $b_{k, p, s}(x)$. This has analogous properties for each operator $\alpha=0,1,2,3$ and we confine the detailed discussion to the case $\alpha=3$, stating the generalization to other values of $\alpha$ when appropriate.

Following the definition of $h_{p+1}(x)$ in Section 2 we find successively that

$$
\begin{aligned}
& h_{p+1}^{[3]}(m x)=\frac{1}{2}\left[g_{2 p+3}\left(m x, \frac{1}{4}\right)-g_{2 p+3}\left(m x, \frac{3}{4}\right)\right] \\
& (5.1)=\frac{1}{2}\left[\bar{B}_{2 p+3}\left(\frac{1}{4}-m x\right)-B_{2 p+3}\left(\frac{1}{4}\right)-\bar{B}_{2 p+3}\left(\frac{3}{4}-m x\right)+B_{2 p+3}\left(\frac{3}{4}\right)\right] /(2 p+3) ! \\
& \quad=\frac{1}{2^{2 p+4}(2 p+2) !}\left[E_{2 p+2}\left(\frac{1}{2}\right)-\bar{E}_{2 p+2}\left(\frac{1}{2}-2 m x\right)\right]
\end{aligned}
$$

where we have used (2.6) and standard relations between the Bernoulli and Euler polynomials. The periodic Euler polynomial $E_{2 p+2}\left(\frac{1}{2}-2 m x\right)$ is a polynomial spline function. This has "joints" at values of $x$ for which $\frac{1}{2}-2 m x$ is an integer. At these points the function and its first $2 p+1$ derivatives are continuous, but there is a discontinuity in its $(2 p+2)$-th derivative. Between neighboring joints the function is a polynomial of degree $2 p+2$ in $x$. These joints occur at the points $x=\frac{2 n+1}{4 m}(n=0,1,2, \ldots)$ which are precisely the points at which function evaluations are required to calculate $P^{[m, 3]} f(x)$.

The function $b_{k, p, s}(x)$ is a linear combination of different functions $h_{p+1}\left(m_{j} x\right)$, $m_{i}$ taking values $m_{k}, m_{k+1}, \ldots, m_{k+p}$, given by

$$
b_{k, p, s}(x)=\sum_{j=k}^{k+p} \mu_{k, p, s, j} a_{j}^{p+1} h_{p+1}\left(m_{j} x\right) .
$$

Using simple properties of the van der Monde matrix it may be shown that none of the coefficients of $h_{p+1}\left(m_{i} x\right)$ are zero. Consequently we are able to state the following theorem; 
Theorem 3. The function $b_{k, t, s}^{[\alpha]}(x)$ is a spline polynomial of degree $d$. Joints occur at each value of $x$ for which any of the $p+1$ operators $p^{\left[m_{j}, \alpha\right]} f(x) j=$ $k, k+1, \ldots, k+p$ require function evaluation. At these points, $b[\alpha], p, s)$ together with its first $d-1$ derivatives are continuous, but its $d$-th derivative is not continuous. Here $d=2 p+2(\alpha=0,1,3)$ and $d=2 p+4(\alpha=2)$.

In principle (5.2) and (5.1) constitute a method for calculating individual values of $b_{k, p, s}(x)$ in terms of tabulated functions. Such expressions seem to be too involved to be useful in any analytical attempt to show that $b_{k, p, s}(x)$ is of definite sign.

However, a relatively simple expression results if we restrict our attention to the domain of $x$ between the end of the interval and the nearest joint. This is because, in this interval $\bar{E}_{2 p+2}\left(\frac{1}{2}-2 m_{j} x\right)$ coincides with $E_{2 p+2}\left(\frac{1}{2}-2 m_{j} x\right)$ for all $m_{j}$. We use the standard expansion

$$
E_{2 p+2}\left(\frac{1}{2}\right)-E_{2 p+2}\left(\frac{1}{2}+2 m x\right)=-\sum_{l=0}^{p}\left(\begin{array}{c}
2 p+2 \\
2 l
\end{array}\right) E_{2 l}\left(\frac{1}{2}\right)(2 m x) .{ }^{2 p+2-2 t} .
$$

Substituting this into (5.1), substituting (5.1) into (5.2) and remembering that $a_{j}=1 / m_{j}^{2}$, we find

$$
b_{k, p, s}=-\sum_{l=0}^{p} \frac{E_{2 l}\left(\frac{1}{2}\right) x^{2 p+2-2 l}}{2^{2 l+2}(2 p+2-2 l) !}\left[\sum_{j=k}^{k+p} \mu_{k, p, s, j} a_{j}^{l}\right], \quad x<\bar{x}_{k, p}
$$

where $\bar{x}_{k, p}$, the location of the first point for function evaluation, is

$$
\bar{x}_{k, p}=\frac{1}{4 \max _{k \leq i \leq k+p} m_{i}} .
$$

In view of (4.2), the expression in square brackets may be replaced by $\delta_{s, l}$ and subsequently the sum over $l$ may be replaced by the term having $l=s$. Since

$$
E_{2 s}\left(\frac{1}{2}\right)=2^{-2 s} E_{2 s}
$$

we find

$$
b_{k, p, s}(x)=-\frac{E_{2 s} x^{2 p+2-2 s}}{2^{4 s+2}(2 p+2-2 s) !(2 s) !}, \quad|x| \leqq \bar{x}_{k, p}
$$

The point of interest about this result is that it depends upon $k$ and $p$ and the maximum mesh ratio, but is independent of all the other mesh ratios.

We state this result and the corresponding result for $\alpha=0$.

Theorem 4. If $x_{k, p}^{[\alpha]}$ is the point for function evaluation nearest the origin required in the calculation of $T_{k, p, s}$ then

where

$$
b_{k, p, s}^{[\alpha]}(x)=c_{p, s}^{[\alpha]} x^{2 p+2-2 s}, \quad x \leqq x_{k, p}^{[\alpha]}
$$

$$
\begin{aligned}
& c_{p, s}^{[0]}=-\left(1-2^{1-2 s}\right) B_{2 s} l((2 p+2-2 s) !(2 s) !) \\
& c_{p, s}^{[3]}=-E_{2 s} /\left(4^{2 s+1}(2 p+2-2 s) !(2 s) !\right) .
\end{aligned}
$$

Particular examples of this may be used in the proofs of Lemmas 4 and 5 in Section 6. 


\section{The Functions $b_{0, p, s}(x)$ Corresponding to $m_{i}=i+1$}

In this section we deal exclusively with the mesh ratios

$$
\begin{aligned}
& m_{i}=i+1, \quad i=0,1,2, \ldots, \\
& a_{i}=1 /(i+1)^{2} .
\end{aligned}
$$

Romberg integration is traditionally carried out using mesh ratios $m_{i}=2^{i}$, $i=0,1,2, \ldots$. This results in a rapid growth of the number of function evaluations. Significantly fewer evaluations are required to achieve comparative truncation error if mesh ratios $m_{i}=i+1, i=0,1,2, \ldots$ are used. However, as Bauer, et al. [2] point out, Romberg integration with these mesh ratios can be numerically unstable; that is, round off errors in the first column of the table are amplified in later columns.

Although this instability is often apparent in practice, it is not excessive in the first few columns. It is a simple matter to estimate an upper bound on the error due to the instability in any column and this may be less than the tolerance required. If not, higher precision arithmetic may be used, its cost being offset by the smaller number of function evaluations required.

In the application to the computation of Fourier coefficients [4], the operators $P^{[m, \alpha]}$ play a dual role. They are used both directly as terms in certain asymptotic expansions and indirectly in the approximation of $I_{s}$ as described here. In the former role they are required for all $m=1,2,3, \ldots$ and it is natural to consider using the same values in the later role.

The results of this section apply to the function $b_{0, p, s}(x)$ and consequently give information relating to the "main diagonal plane" terms of the three dimensional Romberg Table. We prove the following Theorem.

Theorem 5. For $m_{i}=i+1$ and $\alpha=0,1,2,3$ the functions $b_{0, p, s}(x)$ are all of definite sign.

The proof of this theorem is lengthy and we divide it up into several Lemmas.

\section{Lemma 1.}

$$
b_{0, p, s}(x)=2(-1)^{s+1} \sum_{q=1}^{p+1} \frac{4 q((q-1) !)^{2}}{2 q !} \sigma_{0, q-2, s-p-1+q} \sum_{t=1}^{\infty} \frac{N_{r} \sin ^{2 q} \pi r x}{(2 \pi r)^{2 p+2}}
$$

where $\sigma_{0, l, m}(0 \leqq m \leqq l+1)$ are the elementary symmetric functions defined by (4.4),

$$
\begin{aligned}
\sigma_{0,-1,0} & =1 \\
\sigma_{0, l, m} & =0, \quad m<0,
\end{aligned}
$$

and the number $N_{r}$ which depends on the operator is given by (2.15).

If we substitute expression (2.14) for $h_{p+1}\left(m_{j} x\right)$ into the definition (4.10) of $b_{0, p, s}(x)$ we find

$$
b_{0, p, s}(x)=-4 \sum_{r=1}^{\infty} \frac{N_{r}}{(2 \pi r)^{2 p+2}} \sum_{j=0}^{p}(-1)^{p} \mu_{0, p, s, j} a_{j}^{p+1} \sin ^{2} \pi r m_{j} x
$$


where the interchange in the order of the summation operators is valid. We now consider relation (4.4) in the case $k=0, p=q-2$ and $y=1 / m^{2}$. Using the values of $a_{i}$ and $m_{i}$ given in (6.1) and (6.2), a rearrangement of this Eq. (4.4) gives

$$
\prod_{i=0}^{q-2}\left(m^{2}-m_{t}^{2}\right)=((q-1) !)^{2} \sum_{i=0}^{q-1} \sigma_{0, q-2, l}(-1)^{l} m^{2(q-1-l)}
$$

If $m$ is an integer, less than or equal to $p+1, \sin ^{2} m \theta$ may be expressed as a polynomial of degree $p+1$ or less in $\sin ^{2} \theta$. This formula may be written

$$
\sin ^{2} m \theta=\sum_{q=1}^{p+1} \frac{(-1)^{q-1}}{2} \frac{4^{q}}{2 q !}\left(m^{2}-0^{2}\right)\left(m^{2}-1^{2}\right) \ldots\left(m^{2}-(q-1)^{2}\right) \sin ^{2 q} \theta .
$$

We may substitute into (6.7) expression (6.6) for the main part of each term in the sum. We may set $\theta=\pi r x$ in (6.7) and substitute for $\sin ^{2} \pi r m_{j} x$ in (6.5). This gives

$$
\begin{aligned}
b_{0, p, s}(x)= & -2 \sum_{r=1}^{\infty} \frac{N_{r}}{(2 \pi r)^{2 p+1}} \sum_{j=0}^{p} \sum_{q=1}^{p+1} \sum_{l=0}^{q-1} \frac{(-1)^{q-1} 4^{q}((q-1) !)^{2}}{2 q !} \sin ^{2 q} \pi r x \\
& \cdot \mu_{0, p, s, i} a_{j}^{p+1-q+l} \sigma_{0, q-2, l}(-1)^{l} .
\end{aligned}
$$

This expression can be simplified. Since $p+1-q+l$ necessarily lies between 0 and $p$ we may use (4.2) to replace the sum over $j$ and the two terms which involve the suffix $j$ by $\delta_{s, p+1-q+l}$. This factor reduces the sum over $l$ to the single term obtained by setting $l=s+q-p-1$. After introducing these simplications the expression on the right hand side of $(6.8)$ reduces to the expression on the right hand side of (6.3), which establishes Lemma 1.

We see that apart from the sum over $r$, the sign of each term in (6.3) is known. (The quantities $\sigma_{0, l, m}$ being the elementary symmetric functions of the positive numbers $a_{0}, a_{1}, \ldots, a_{l}$ are all positive.) Thus if the expressions

$$
S_{p+1, q}^{[\alpha]}(x)=\sum_{r=1}^{\infty} \frac{N_{r}^{[\alpha]} \sin ^{2 q} \pi r x}{(2 \pi r)^{2 p+2}}, \quad q=1,2, \ldots, p+1
$$

were of definite sign for all $x$, this sign being the same for all $q$, then it would follow from (6.3) that $b_{0, p, s}^{[\alpha]}(x)$ is of definite sign.

\section{Lemma 2.}

$$
\begin{aligned}
& S_{p+1, q}^{[1]}(x) \leqq 0, \\
& S_{p+1, q}^{[2]}(x) \geqq 0, \quad q=1,2, \ldots, p+1 .
\end{aligned}
$$

Proof. From the list of values of $N_{r}^{[\alpha]}$ in (2.15) we see that $N_{r}^{[1]} \leqq 0$ (and $N_{r}^{[2]} \geqq 0$ ) for all $r$. Thus $S_{p, q}^{[\alpha]}(x)$ is simply the sum of negative (or positive) terms and is accordingly of the indicated definite sign.

\section{Lemma 3.}

$$
S_{p+1, q}^{[0]}(x) \geqq 0, \quad q=1,2, \ldots, p
$$


Proof. Using standard elementary manipulation, it follows that

$$
\begin{aligned}
S_{p+1, q}^{[0]}(x) & =\sum_{r=1}^{\infty} \frac{(-1)^{r-1} \sin ^{2 q}(\pi r x)}{(2 \pi r)^{2 p+2}} \\
& =\sum_{r=1}^{\infty} \frac{\sin ^{2} q(\pi r x)}{(2 \pi r)^{2 p+2}}-2 \sum_{r \text { even }} \frac{\sin ^{2} q(\pi r x)}{(2 \pi r)^{2 p+2}} \\
& =\sum_{r=1}^{\infty} \frac{\sin ^{2} q(\pi r x)}{(2 \pi r)^{2} p+2}-\frac{2}{2^{2 p+2}} \sum_{r=1}^{\infty} \frac{\sin ^{2} q(2 \pi r x)}{(2 \pi r)^{2 p+2}} \\
& =\sum_{r=1}^{\infty} \frac{\sin ^{2} q(\pi r x)}{(2 \pi r)^{2 p+2}}\left\{1-\frac{1}{2^{2 p+1-2 q}} \cos ^{2 q}(\pi r x)\right\} .
\end{aligned}
$$

Since $q \leqq p$, this is the sum of positive terms. This establishes Lemma 3 .

\section{Lemma $4^{\star}$.}

$$
S_{p+1, p+1}^{[0]}(x)=\sum_{r=1}^{\infty}(-1)^{r-1}\left(\frac{\sin \pi r x}{2 \pi r}\right)^{2 p+2} \geqq 0
$$

\section{Lemma $5^{\star}$.}

$$
S_{p+1, q}^{[3]}(x)=\sum_{\text {odd } r}^{\infty} \frac{(-1)^{(r+1) / 2} \sin ^{2 q} \pi r x}{(2 \pi r)^{2 p+2}} \geqq 0, \quad q=1,2, \ldots, p+1 .
$$

Lemmas $2-5$ taken together show that the sum $S_{p+1, q}^{[\alpha]}(x)$ which occurs in (6.3) is of the same sign for all $x$ and $q$. Since the other terms in (6.3) are of definite sign, it follows that $b_{0, p, s}(x)$ is of definite sign and that Theorem 5 is valid.

\section{Conclusion}

In the original presentation of the application of the Neville algorithm to numerical quadrature, Romberg [6] confined his attention to the trapezoidal rules $R^{\left[m_{i}, 1\right]} f(x)$ using mesh ratios $m_{i}=1,2,4,8, \ldots$ to evaluate approximations to the integral $I f(x)$. A full discussion of Romberg Integration is given by Bauer, Rutishauser and Stiefel [2] who obtain an expression for the truncation error in the standard form

$$
T_{p}^{(k)}-I f(x)=c_{r, p} f^{(2 p+2)}(\xi), \quad 0 \leqq \xi \leqq 1 .
$$

Bulirsch discusses the generalization of the algorithm to take into account an arbitrary choice of mesh ratios $m_{i}$. He also obtains results corresponding to (7.1) for two particular sets of mesh ratios, $m_{i}=i+1$ and $m_{i}=3 \times 2^{(i-1) / 2}(i$ odd $)$, $m_{i}=2^{(i+2) / 2}(i$ even).

In this paper we make further generalizations. These include replacing the operator $R^{[m, 1]}$ by any linear combination of off set trapezoidal rules which have an even expansion in inverse powers of $m$; also we consider the calculation of

* The author's own proofs of Lemmas 4 and 5 are correct but inelegant. Consequently Lemma 4 was submitted as a problem to SIAM Review (9, April 1967, problem 67-6, page 250). The solution published, SIAM Review 10, pp. 226-7 is elegant but incorrect. Subsequently Dr. R. Askey has pointed out to the authors an elegant and correct proof based on Turán's Theorem. This will be included in work to be published by Dr. Askey and Dr. J. Fitch. 
the integrals of derivatives of $f(x)$ such as $I f^{(s)}(x)$. The Neville algorithm is extended to calculate these quantities for any choice of mesh ratios.

We also consider the truncation error. In the case of four simple operators we show that the error corresponding to the mesh ratio choice $m_{i}=i+1$ is of the form (7.1) above.

One feature of these results is that the problem is simple to pose and the result is simple to state. The intervening proof is lengthy and arduous though it involves only elementary analysis. These proofs do not seem to generalize in any obvious way to similar related problems.

\section{References}

1. Abramowitz, M., Stegun, I. A.: Handbook of mathematical functions. New York: Dover Inc. (1965).

2. Bauer, F. L., Rutishauser, H., Stiefel, E.: New aspects in numerical quadrature. Proc. Symposia on Applied Mathematics 15, 199-218 (1963).

3. Bulirsch, R.: Bemerkungen zur Romberg-Integration. Numer. Math. 6, 6-16 (1966).

4. Lyness, J. N.: The calculation of Fourier coefficients. SIAM J. Numer. Anal. 4, $301-315(1967)$.

5. -, Moler, C. B.: Van der Monde systems and numerical differentiation. Numer. Math. 8, 458-464 (1966).

6. Romberg, W.: Vereinfachte numerische Integration. Det. Kong. Nomke Videnskaber Selskab Forhandlinger, Bd 28, Nr. 7, Trondheim (1955).

7. Stetter, H.: Numerical approximation of Fourier transforms. Numer. Math. 8, $235-249$ (1966).

J. N. Lyness

C. B. Moler

Institut für angewandte Mathematik

der Eidgen. Techn. Hochschule

CH 8000 Zürich 